\title{
EXPONENTIAL SUMS OVER PRIMES IN SHORT INTERVALS AND AN APPLICATION TO THE WARING-GOLDBACH PROBLEM
}

\author{
BINGRONG HUANG
}

\begin{abstract}
Let $\Lambda(n)$ be the von Mangoldt function, $x$ real and $2 \leq y \leq x$. This paper improves the estimate on the exponential sum over primes in short intervals

$$
S_{k}(x, y ; \alpha)=\sum_{x<n \leq x+y} \Lambda(n) e\left(n^{k} \alpha\right)
$$

when $k \geq 3$ for $\alpha$ in the minor arcs. And then combined with the Hardy-Littlewood circle method, this enables us to investigate the Waring-Goldbach problem of representing a positive integer $n$ as the sum of $s k$ th powers of almost equal prime numbers, which improves the results in Wei and Wooley [14].
\end{abstract}

\section{Contents}

1. Introduction

2. Auxiliary results

3. Type I estimate

4. Type II estimate

5. Proof of Theorem 1

6. Proof of Theorem 2

References

\section{INTRODUCTION}

Let $\Lambda(n)$ be the von Mangoldt function, $k \geq 1$ an integer, $x$ real and $2 \leq y \leq x$. The estimate of the exponential sum over primes in short intervals

$$
S_{k}(x, y ; \alpha)=\sum_{x<n \leq x+y} \Lambda(n) e\left(n^{k} \alpha\right)
$$

was first studied by I. M. Vinogradov [13] in 1939 with his elementary method. Since then this topic has attracted the interest of quite a number of authors. These sums arise naturally and play important roles when solving the Waring-Goldbach problems in short intervals by the circle method. In particular, the case $k=1$, i.e., the linear exponential sum over primes in short intervals, was studied quite extensively, because

Date: May 31, 2016.

Key words and phrases. Exponential sums, von Mangoldt function, short intervals, Waring-Goldbach problem. 
of its applications to the study of the Goldbach-Vinogradov theorem with three almost equal prime variables (see [15] and the references therein).

For the case $k=2$, Liu and Zhan [10] first established a non-trivial estimate of $S_{2}(x, y ; \alpha)$ for all $\alpha$ and all published results before their result are valid only for $\alpha$ in a very thin subset of $[0,1]$. In [11], Lü and Lao improved the results in [10] to be as good as what was previously derived from the Generalized Riemann Hypothesis.

In this paper we deal with $S_{k}(x, y ; \alpha)$ in the general case $k \geq 3$. Let $y=x^{\theta}$ with $3 / 4<\theta \leq 1$. We set

$$
P=x^{2 K \delta}, \quad \text { and } \quad Q=x^{k-2} y^{2} P^{-1}
$$

where

$$
K=2 t_{k}\left(t_{k}+2\right), \quad t_{k}=k(k-1)
$$

are defined as in [14, equations (1.2) and (2.1)] and $\delta$ is a positive parameter which may depend on $k$. By Dirichlet's theorem on Diophantine approximations, every real number $\alpha$ has a rational approximation $a / q$, where $a$ and $q$ are integers subject to

$$
1 \leq q \leq Q, \quad(a, q)=1, \quad|q \alpha-a| \leq 1 / Q .
$$

We denote by $\mathfrak{M}$ the union of the major arcs

$$
\mathfrak{M}(q, a)=\left\{\alpha \in[0,1):|q \alpha-a| \leq Q^{-1}\right\},
$$

with $0 \leq a \leq q \leq P$ and $(a, q)=1$, and let $\mathfrak{m}=[0,1) \backslash \mathfrak{M}$ for the set of minor arcs complementary to the set $\mathfrak{M}$. In Liu and Zhan [9] and Huang and Wang [3], they handle with $S_{k}(x, y ; \alpha)$ for all $\alpha \in[0,1]$ by dividing this into several parts and then estimating them by different methods. The work of Liu, Lü and Zhan [8] is focused on the major arc behavior (hence, the use of multiplicative methods) and provides a major arc estimate that complements the "true minor arc bound" from an earlier paper of Liu and Zhan [9]. Kumchev [7] and Wei and Wooley [14] focus on the minor arcs. The method of Kumchev [7] is a variant of his earlier results on long sums [6] that avoids the use of sieve methods and relies on Heath-Brown's identity instead. Recently, Wei and Wooley [14] gives a substitute for a Weyl-type estimate for $S_{k}(x, y ; \alpha)$, which makes use of Daemen's estimates in [1] via a bilinear form treatment motivated by analogous arguments making use of Vinogradov's mean value theorem. They establish an estimate of Weyl-type that delivers non-trivial estimates throughout the set of minor arcs $\mathfrak{m}$ provided that $\theta$ is a real number with $5 / 6<\theta<1$. We first state our main result for exponential sums.

Theorem 1. Let $k \geq 3$. Let $\theta$ be a real number with $3 / 4<\theta \leq 1$ and suppose that $0<\rho<\rho_{k}(\theta)$, where

$$
\rho_{k}(\theta)=\min \left\{\frac{\sigma_{k}(\theta-3 / 4)}{8}, \delta\right\},
$$

with $\sigma_{k}=1 /\left(2 t_{k}\right)$. Then, for any fixed $\varepsilon>0$, we have

$$
S_{k}(x, y ; \alpha) \ll y^{1-\rho+\varepsilon}+\frac{y x^{\varepsilon}}{\left(q+y^{2} x^{k-2}|q \alpha-a|\right)^{1 /(2 k)}} .
$$


Remark 1. In [2], we asserts the strong orthogonality between the Möbius function and nonlinear exponential functions in short intervals. More specifically, if $k \geq 3$ being fixed and $y \geq x^{1-1 / 4+\varepsilon}$, then for any $A>0$, we have

$$
\sum_{x<n \leq x+y} \mu(n) e\left(n^{k} \alpha\right) \ll y(\log y)^{-A}
$$

uniformly for $\alpha \in \mathbb{R}$.

To prove Theorem 1, we first use Vaughan's identity to divide this to two types of sums, and then estimate the exponential sums of type I and type II respectively (see \$5). The exponential sums of type I will be estimated in 93 , and the other one will be treated in $\$ 4$, To do these we mainly follow the method of Kumchev [7], and combine with the results in Daemen [1] (see §2, 41).

In the last section, we apply the circle method to give an application of this exponential sum to Waring-Goldbach problem in short intervals.

A formal application of the circle method suggests that whenever $s$ and $k$ are natural numbers with $s \geq k+1$, then all large integers $n$ satisfying appropriate local conditions should be represented as the sum of $s k$-th powers of prime numbers. With this expectation in mind, consider a natural number $k$ and prime $p$, take $\tau=\tau(k, p)$ to be the integer with $p^{\tau} \mid k$ but $p^{\tau+1} \nmid k$, and the define $\eta=\eta(k, p)$ by putting $\eta(k, p)=\tau+2$, when $p=2$ and $\tau>0$, and otherwise $\eta(k, p)=\tau+1$. We then define $R=R(k)$ by putting $R(k)=\prod p^{\eta}$, where the product is taken over primes $p$ with $(p-1) \mid k$. Write $X=(n / s)^{1 / k}$. We say that the exponent $\Delta_{k, s}$ is admissible when, provided that $\Delta$ is a positive number with $\Delta<\Delta_{k, s}$, then for all sufficiently large positive integers $n$ with $n \equiv s(\bmod R)$, the equation

$$
p_{1}^{k}+p_{2}^{k}+\cdots+p_{s}^{k}=n
$$

has a solution in prime numbers $p_{j}$ satisfying $\left|p_{j}-X\right| \leq X^{1-\Delta}(1 \leq j \leq s)$. We refer the reader to [14] for more details.

Together the circle method used in [14] with our estimate of exponential sums in Theorem 1, we show that there are larger admissible exponents $\Delta_{k, s}$ as soon as $s>2 t_{k}$.

Theorem 2. Let $s$ and $k$ be integers with $k \geq 3$ and $s>2 t_{k}$. Suppose that $\varepsilon>0$, that $n$ is a sufficiently large number satisfying $n \equiv s(\bmod R)$, and write $X=(n / s)^{1 / k}$. Then the equation $n=p_{1}^{k}+p_{2}^{k}+\cdots+p_{s}^{k}$ has a solution in prime numbers $p_{j}$ with $\left|p_{j}-X\right| \leq$ $X^{19 / 24+\varepsilon}(1 \leq j \leq s)$.

In [14], Wei and Wooley gave the same exponent $\frac{19}{24}$ for the case $k=2$. They remark that since $\frac{19}{24}=\frac{1}{2}\left(1+\frac{7}{12}\right)$, this exponent is in some sense half way between the trivial exponent 1 and the exponent $\frac{7}{12}$ that, following the work of Huxley [4], represents the effective limit of our knowledge concerning the asymptotic distribution of prime numbers in short intervals.

By the same argument in [14, §9], we obtain the following almost-all result. (The history of this kind of problem can be seen in [14, §1].) 
Theorem 3. Let $s$ and $k$ be integers with $k \geq 3$ and $s>t_{k}$. Suppose that $\varepsilon>0$. Then for almost all positive integers $n$ with $n \equiv s(\bmod R)$, (and, in case $k=3$ and $s=7$, satisfying also $9 \nmid n)$, the equation $n=p_{1}^{k}+p_{2}^{k}+\cdots+p_{s}^{k}$ has a solution in prime numbers $p_{j}$ with $\left|p_{j}-X\right| \leq X^{19 / 24+\varepsilon}(1 \leq j \leq s)$, where $X=(n / s)^{1 / k}$.

Notation. Throughout the paper, the letter $\varepsilon$ denotes a sufficiently small positive real number which may be different at each occurrence. For example, we may write $x^{\varepsilon} \ll y^{\varepsilon}$. Any statement in which $\varepsilon$ occurs holds for each positive $\varepsilon$, and any implied constant in such a statement is allowed to depend on $\varepsilon$. The letter $p$, with or without subscripts, is reserved for prime numbers. In addition, as usual, $e(z)$ denotes $e^{2 \pi i z}$. We write $(a, b)=\operatorname{gcd}(a, b)$, and we use $m \sim M$ as an abbreviation for the condition $M<m \leq 2 M$.

\section{Auxiliary Results}

The following lemma is useful to give an estimate for exponential sums of type I and type II which is an improvement of [7, Lemma 2.2].

Lemma 1. Let $k \geq 3$ be an integer and $\gamma \geq 3$ be a real number. Let $0<\rho \leq \sigma_{k} / \gamma$, where $\sigma_{k}=1 /\left(2 t_{k}\right)$. Suppose that $y \leq x$, and $y \geq x^{\frac{\gamma}{2 \gamma-\sigma_{k}-1}}$. Then either

$$
\sum_{x<n \leq x+y} e\left(n^{k} \alpha\right) \ll y^{1-\rho+\varepsilon}
$$

or there exist integers $a$ and $q$ such that

$$
1 \leq q \leq y^{k \rho}, \quad(a, q)=1, \quad|q \alpha-a| \leq x^{1-k} y^{k \rho-1},
$$

and

$$
\sum_{x<n \leq x+y} e\left(n^{k} \alpha\right) \ll y^{1-\rho+\varepsilon}+\frac{y}{\left(q+y x^{k-1}|q \alpha-a|\right)^{1 / k}} .
$$

Proof. Take

$$
P_{0}=y^{1 / \gamma} \quad \text { and } \quad Q_{0}=x^{k-2} y^{2} / P_{0} .
$$

By Dirichlet's theorem on Diophantine approximation, there exists integers $a$ and $q$ with

$$
1 \leq q \leq Q_{0}, \quad(a, q)=1, \quad|q \alpha-a| \leq 1 / Q_{0} .
$$

When $q>P_{0}$, we rewrite the sum on the left of (2.1) as

$$
\sum_{1 \leq n \leq z} e\left(\alpha_{k} n^{k}+\alpha_{k-1} n^{k-1}+\cdots+\alpha_{0}\right)
$$

where $z \leq y$ and $\alpha_{j}=\left(\begin{array}{l}k \\ j\end{array}\right) \alpha u^{k-j}$, with $u$ a fixed integer. Hence, it follows from the argument underlying the proof of [1, eq. (3.5)] and [14, eq. (4.23)] that

$$
\sum_{x<n \leq x+y} e\left(n^{k} \alpha\right) \ll y P_{0}^{-1 /\left(2 t_{k}\right)+\varepsilon} \ll y^{1-\rho+\varepsilon} .
$$


When $q \leq P_{0}$, from [1, eq. (5.1)-(5.5) and $\left.\S 6\right]$, we deduce

$$
\sum_{1 \leq n \leq y} e\left(\alpha_{k} n^{k}+\alpha_{k-1} n^{k-1}+\cdots+\alpha_{0}\right) \ll \frac{y}{\left(q+y x^{k-1}|q \alpha-a|\right)^{1 / k}}+\Delta,
$$

where

$$
\Delta \ll P_{0}^{1 / 2+\varepsilon}\left(1+\frac{P_{0} x^{k}}{x^{k-2} y^{2}}\right)^{1 / 2} \ll P_{0}^{1+\varepsilon} x / y \ll y^{1-\rho+\varepsilon},
$$

provided that $y \geq x^{\frac{\gamma}{2 \gamma-\sigma_{k}-1}}$. Thus, at least one of (2.1) and (2.3) holds. The lemma follows on noting that when conditions (2.2) fail, inequality (2.1) follows from (2.3).

The next lemma gives some inequalities which will be used in the following sections.

Lemma 2. We have

$$
\sum_{n \sim N}\left(r, n^{k}\right)^{1 / k} \leq N \tau(r)
$$

where $\tau(r)$ is the divisor function; and for any $\varepsilon>0$, we have

$$
\sum_{\substack{n \sim N \\(n, h)=1}}(r, R(n, h))^{1 / k} \ll N r^{\varepsilon}+r^{1 / k+\varepsilon},
$$

where $R(n, h)=\left((n+h)^{k}-n^{k}\right) / h$.

Proof. We have

$$
\sum_{n \sim N}\left(r, n^{k}\right)^{1 / k} \leq \sum_{n \sim N}(n, r) \leq \sum_{d \mid r} d \sum_{\substack{n \sim N \\ d \mid n}} 1 \leq N \tau(r) .
$$

To prove (2.7), see the inequality (3.11) in Kawada and Wooley [5].

\section{Type I estimate}

The following proposition treats the exponential sums of type I which is an improvement of [3, Lemma 8]

Proposition 1. Let $k \geq 3$ be an integer and $\gamma \geq 3$ be a real number. Let $0<\rho<$ $\min \left\{\sigma_{k} /(2 \gamma), \delta\right\}$, with $\sigma_{k}=1 /\left(2 t_{k}\right)$. Suppose that $\alpha$ is real that there exist integers $a$ and $q$ such that (1.4) holds with $Q$ given by (1.2). Let $\xi(m) \ll m^{\varepsilon}$, and define

$$
\mathcal{T}_{1}=\sum_{m \sim M} \xi(m) \sum_{x<m n \leq x+y} e\left((m n)^{k} \alpha\right)
$$

Then

provided that

$$
\mathcal{T}_{1} \ll y^{1-\rho+\varepsilon}+\frac{y x^{\varepsilon}}{\left(q+y x^{k-1}|q \alpha-a|\right)^{1 / k}}
$$

$$
M \ll y\left(\frac{y}{x}\right)^{\frac{\gamma}{\gamma-\sigma_{k}-1}}, \quad M \ll y x^{-\gamma \rho / \sigma_{k}}, \quad M^{2 k} \ll y x^{k-1-2 k \rho} .
$$


Proof. Set

$$
S_{m}=\sum_{X<n \leq X+Y} e\left(m^{k} n^{k} \alpha\right)
$$

where $X=x / m, Y=y / m$, with $m \sim M$. Define $\nu$ by $Y^{\nu}=x^{\rho} L^{-1}$. Note that, by (3.1), we have

$$
\nu<\sigma_{k} / \gamma
$$

We denote by $\mathcal{M}$ the set of integers $m \sim M$, for which there exist integers $b_{1}$ and $r_{1}$ with

$$
1 \leq r_{1} \leq Y^{k \nu}, \quad\left(b_{1}, r_{1}\right)=1, \quad\left|r_{1} m^{k} \alpha-b_{1}\right| \leq X^{1-k} Y^{k \nu-1} .
$$

We apply Lemma 1 to the summation over $n$ and get

$$
S_{m} \ll Y^{1-\nu+\varepsilon}+\frac{Y}{\left(r_{1}+Y X^{k-1}\left|r_{1} m^{k} \alpha-b_{1}\right|\right)^{1 / k}},
$$

for $m \in \mathcal{M}$. So

$$
\mathcal{T}_{1} \ll \sum_{m \sim M}|\xi(m)| Y^{1-\nu+\varepsilon}+\sum_{m \in \mathcal{M}} \frac{|\xi(m)| Y}{\left(r_{1}+Y X^{k-1}\left|r_{1} m^{k} \alpha-b_{1}\right|\right)^{1 / k}} .
$$

Then, we have

$$
\mathcal{T}_{1} \ll y^{1-\rho+\varepsilon}+T_{1}(\alpha)
$$

where

$$
T_{1}(\alpha)=\sum_{m \in \mathcal{M}} \frac{|\xi(m)| Y}{\left(r_{1}+Y X^{k-1}\left|r_{1} m^{k} \alpha-b_{1}\right|\right)^{1 / k}} .
$$

We apply Dirichlet's theorem on Diophantine approximation to find integers $b$ and $r$ with

$$
1 \leq r \leq x^{-k \rho} Y X^{k-1}, \quad(b, r)=1, \quad|r \alpha-b| \leq x^{k \rho} Y^{-1} X^{1-k} .
$$

By (3.1), (3.2) and (3.3), we have

$$
\begin{aligned}
\left|b_{1} r-b m^{k} r_{1}\right| & =\left|r\left(b_{1}-r_{1} m^{k} \alpha\right)+r_{1} m^{k}(r \alpha-b)\right| \\
& \leq x^{-k \rho} Y X^{k-1} X^{1-k} Y^{k \nu-1}+Y^{k \nu}(2 M)^{k} x^{k \rho} Y^{-1} X^{1-k} \\
& \ll L^{-k}+M^{2 k} L^{-k} x^{2 k \rho-k+1} y^{-1} \ll L^{-k}<1,
\end{aligned}
$$

whence

Thus, by Lemma 2, we have

$$
\frac{b_{1}}{r_{1}}=\frac{m^{k} b}{r}, \quad r_{1}=\frac{r}{\left(r, m^{k}\right)}
$$

$$
\begin{aligned}
T_{1}(\alpha) & \leq \sum_{m \in \mathcal{M}} \frac{|\xi(m)| y M^{-1} r_{1}^{-1 / k}}{\left(1+Y X^{k-1}\left|m^{k} \alpha-m^{k} b / r\right|\right)^{1 / k}} \\
& \ll \frac{y M^{-1+\varepsilon}}{\left(1+y x^{k-1}|\alpha-b / r|\right)^{1 / k}} \sum_{m \sim M}\left(\frac{r}{\left(r, m^{k}\right)}\right)^{-1 / k} \\
& \ll \frac{y x^{\varepsilon}}{\left(r+y x^{k-1}|r \alpha-b|\right)^{1 / k}} .
\end{aligned}
$$


Recall that $b$ and $r$ satisfy the conditions (3.3). We now consider three cases depending on the size of $r$ and $|r \alpha-b|$.

Case 1: If $r>x^{k \rho}$, then $T_{1}(\alpha) \ll y^{1-\rho+\varepsilon}$;

Case 2: If $r \leq x^{k \rho}$ and $|r \alpha-b|>y^{-1} x^{1-k} x^{k \rho}$, then $T_{1}(\alpha) \ll y^{1-\rho+\varepsilon}$;

Case 3: If $r \leq x^{k \rho}$ and $|r \alpha-b| \leq y^{-1} x^{1-k} x^{k \rho}$, we have

$$
\begin{aligned}
|r a-b q| & =|r(a-q \alpha)+q(r \alpha-b)| \\
& \leq x^{k \rho} \frac{1}{Q}+Q y^{-1} x^{1-k} x^{k \rho} \\
& \leq \frac{x^{k \rho} P}{x^{k-2} y^{2}}+\frac{y x^{k \rho}}{x P} .
\end{aligned}
$$

Since $\rho<\delta$, we have $|r a-b q|<1$, hence

$$
a=b, \quad q=r .
$$

Then

So we prove

$$
T_{1}(\alpha) \ll \frac{y x^{\varepsilon}}{\left(q+y x^{k-1}|q \alpha-a|\right)^{1 / k}} .
$$

$$
\mathcal{T}_{1} \ll y^{1-\rho+\varepsilon}+\frac{y x^{\varepsilon}}{\left(q+y x^{k-1}|q \alpha-a|\right)^{1 / k}} .
$$

Remark 2. Let

$$
\mathcal{T}_{1}^{*}=\sum_{m \sim M} \xi(m) \sum_{x<m n \leq x+y} e\left((m n)^{k} \alpha\right) \log n .
$$

Under the condition of Proposition 1 we have

$$
\mathcal{T}_{1}^{*} \ll y^{1-\rho+\varepsilon}+\frac{y x^{\varepsilon}}{\left(q+y x^{k-1}|q \alpha-a|\right)^{1 / k}} .
$$

Remark 3. One can estimate the exponential sums

$$
\sum_{m_{1} \sim M_{1}} \sum_{m_{2} \sim M_{2}} \xi\left(m_{1}, m_{2}\right) \sum_{x<m_{1} m_{2} n \leq x+y} e\left(\left(m_{1} m_{2} n\right)^{k} \alpha\right)
$$

with some suitable conditions on $M_{1}$ and $M_{2}$ as [7, Lemma 3.2] and [14, Lemma 4.2] did, and then may give a better result than Proposition 1. Since it has no influence on our main results, we will not do it.

\section{Type II estimate}

To prove Theorem 1, we also need to handle the exponential sums of type II. Let $\xi(m)$ and $\eta(n)$ be arithmetic functions satisfying the property that for all natural numbers $m$ and $n$, one has

$$
\xi(m) \ll m^{\varepsilon} \quad \text { and } \quad \eta(n) \ll n^{\varepsilon} \text {. }
$$


Let $M$ and $N$ be positive parameters, and define the exponential sum $\mathcal{T}_{2}=\mathcal{T}_{2}(\alpha ; M)$ by

$$
\mathcal{T}_{2}(\alpha ; M):=\sum_{M<m \leq 2 M} \xi(m) \sum_{x<m n \leq x+y} \eta(n) e\left((m n)^{k} \alpha\right) .
$$

The following proposition gives an estimate for $\mathcal{T}_{2}$ which is an improvement of [7, Lemma $3.1]$

Proposition 2. Let $k, \gamma, \sigma_{k}$ be as in Proposition 1. Let $0<\rho<\min \left\{\sigma_{k} /(8 \gamma), \delta\right\}$. Suppose that $\alpha$ is real that there exist integers $a$ and $q$ such that (1.4) holds with $Q$ given by (1.2). And let $x$ and $y$ be positive numbers with

$$
y=x^{\theta}, \quad \frac{1}{(1-2 \rho)} \frac{3 \gamma-\sigma_{k}-1}{2\left(2 \gamma-\sigma_{k}-1\right)} \leq \theta \leq 1 .
$$

Then

provided that

$$
\mathcal{T}_{2} \ll y^{1-\rho+\varepsilon}+\frac{y x^{\varepsilon}}{\left(q+y^{2} x^{k-2}|q \alpha-a|\right)^{1 /(2 k)}},
$$

$$
x^{1 / 2} \leq M \ll y^{1-2 \rho} .
$$

Proof. Set $N=x / M, X=x / N$ and $Y=y / N=y M / x$. Define $\nu$ by $Y^{\nu}=x^{2 \rho} L^{-1}$. By (4.4), we have

$$
\nu<\sigma_{k} / \gamma
$$

For $n_{1}, n_{2} \leq 2 N$, let

$$
\mathcal{M}\left(n_{1}, n_{2}\right)=\left\{m \in(M, 2 M]: x<m n_{1}, m n_{2} \leq x+y\right\} .
$$

By Cauchy's inequality and an interchange of the order of summation, we have

$$
\mathcal{T}_{2}^{2} \ll y^{1+\varepsilon} M+M x^{\varepsilon} T_{1}(\alpha),
$$

where

$$
T_{1}(\alpha)=\sum_{n_{1}<n_{2}}\left|\sum_{m \in \mathcal{M}\left(n_{1}, n_{2}\right)} e\left(\alpha\left(n_{2}^{k}-n_{1}^{k}\right) m^{k}\right)\right| .
$$

Let $\mathcal{N}$ denote the set of pairs $\left(n_{1}, n_{2}\right)$ with $n_{1}<n_{2}$ and $\mathcal{M}\left(n_{1}, n_{2}\right) \neq \varnothing$ for which there exist integers $b$ and $r$ such that

$$
1 \leq r \leq Y^{k \nu}, \quad(b, r)=1, \quad\left|r\left(n_{2}^{k}-n_{1}^{k}\right) \alpha-b\right| \leq Y^{k \nu-1} X^{1-k} .
$$

Since $N / 2<n_{1}<n_{2} \leq 2 N$ and $\mathcal{M}\left(n_{1}, n_{2}\right) \neq \varnothing$, we have $n_{2}-n_{1} \leq y x^{-1} n_{1}$. Hence $\# \mathcal{N} \ll x y M^{-2}$. In order to handle the inner summation in $T_{1}(\alpha)$, we set

$$
\begin{aligned}
& X_{1}=\max \left\{M, \frac{x}{n_{1}}\right\} \asymp M=\frac{x}{N}=X \\
& Y_{1}=\min \left\{2 M, \frac{x+y}{n_{2}}\right\}-\max \left\{M, \frac{x}{n_{1}}\right\} \ll \frac{y}{N}=Y .
\end{aligned}
$$


If $Y_{1}<X_{1}^{\gamma /\left(2 \gamma-\sigma_{k}-1\right)}$, by (4.3) and (4.4), the contribution to $T_{1}(\alpha)$ is

$$
\ll x y M^{-2} M^{\gamma /\left(2 \gamma-\sigma_{k}-1\right)} \ll y^{2-2 \rho+\varepsilon} M^{-1} .
$$

If $Y_{1} \geq X_{1}^{\gamma /\left(2 \gamma-\sigma_{k}-1\right)}$, since $\nu<\sigma_{k} / d$, we can apply Lemma 11 with $\rho=\nu, x=X_{1}$ and $y=Y_{1}$ to the inner summation in $T_{1}(\alpha)$. We get

$$
T_{1}(\alpha) \ll y^{2-2 \rho+\varepsilon} M^{-1}+T_{2}(\alpha),
$$

where

$$
T_{2}(\alpha)=\sum_{\left(n_{1}, n_{2}\right) \in \mathcal{N}} \frac{Y}{\left(r+Y X^{k-1}\left|r\left(n_{2}^{k}-n_{1}^{k}\right) \alpha-b\right|\right)^{1 / k}} .
$$

We now change the summation variables in $T_{2}(\alpha)$ to

$$
d=\left(n_{1}, n_{2}\right), \quad n=n_{1} / d, \quad h=\left(n_{2}-n_{1}\right) / d .
$$

We obtain

$$
T_{2}(\alpha) \ll \sum_{d h \leq y / M} \sum_{n}^{\prime} \frac{Y}{\left(r+Y X^{k-1}\left|r h d^{k} R(n, h) \alpha-b\right|\right)^{1 / k}},
$$

where $R(n, h)=\left((n+h)^{k}-n^{k}\right) / h$ and the inner summation is over $n$ with $(n, h)=1$ and $(n d,(n+h) d) \in \mathcal{N}$. For each pair $(d, h)$ appearing in the summation on the right side of (4.8), Dirichlet's theorem on Diophantine approximation yields integers $b_{1}$ and $r_{1}$ with

$$
1 \leq r_{1} \leq x^{-2 k \rho} Y X^{k-1}, \quad\left(b_{1}, r_{1}\right)=1, \quad\left|r_{1} h d^{k} \alpha-b_{1}\right| \leq x^{2 k \rho} Y^{-1} X^{1-k} .
$$

As $R(n, h) \leq 4^{k}(N / d)^{k-1}$, combining (4.4), (4.6) and (4.9), we have

$$
\begin{aligned}
\left|b_{1} r R(n, h)-b r_{1}\right| & =\left|r R(n, h)\left(b_{1}-r_{1} h d^{k} \alpha\right)+r_{1}\left(r h d^{k} R(n, h) \alpha-b\right)\right| \\
& \leq r_{1} Y^{k \nu-1} X^{1-k}+r R(n, h) x^{2 k \rho} Y^{-1} X^{1-k} \\
& \leq L^{-k}+4^{k} N^{k-1} x^{2 k \rho} L^{-k} x^{2 k \rho} Y^{-1} X^{1-k}<1 .
\end{aligned}
$$

Hence,

$$
\frac{b}{r}=\frac{b_{1} R(n, h)}{r_{1}}, \quad r=\frac{r_{1}}{\left(r_{1}, R(n, h)\right)} .
$$

Combining (4.8) and (4.10), we obtain

$$
T_{2}(\alpha) \ll \sum_{d h \leq y / M} \frac{Y}{\left(r_{1}+Y X^{k-1} N_{d}^{k-1}\left|r_{1} h d^{k} \alpha-b_{1}\right|\right)^{1 / k}} \sum_{\substack{n \sim N_{d} \\(n, h)=1}}\left(r_{1}, R(n, h)\right)^{1 / k},
$$

where $N_{d}=N / d$. By Lemma 2, we deduce that

$$
T_{2}(\alpha) \ll y^{2} x^{-1+\varepsilon}+T_{3}(\alpha),
$$

where

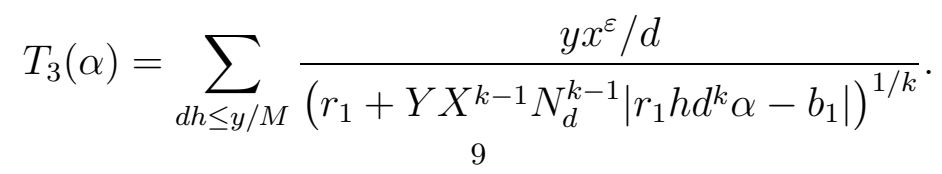


We now write $\mathcal{H}$ for the set of pairs $(d, h)$ with $d h \leq y / M$ for which there exist integers $b_{1}$ and $r_{1}$ subject to

$$
1 \leq r_{1} \leq x^{2 k \rho}, \quad\left(b_{1}, r_{1}\right)=1, \quad\left|r_{1} h d^{k} \alpha-b_{1}\right| \leq x^{-k+1+2 k \rho} Y^{-1} .
$$

We have

$$
T_{3}(\alpha) \ll y^{2-2 \rho+\varepsilon} M^{-1}+T_{4}(\alpha),
$$

where

$$
T_{4}(\alpha)=\sum_{(d, h) \in \mathcal{H}} \frac{y x^{\varepsilon} / d}{\left(r_{1}+Y X^{k-1} N_{d}^{k-1}\left|r_{1} h d^{k} \alpha-b_{1}\right|\right)^{1 / k}} .
$$

For each $d \leq y / M$, Dirichlet's theorem on Diophantine approximation yields integers $b_{2}$ and $r_{2}$ with

$$
1 \leq r_{2} \leq x^{k-1-2 k \rho} Y / 2, \quad\left(b_{2}, r_{2}\right)=1, \quad\left|r_{2} d^{k} \alpha-b_{2}\right| \leq 2 x^{-k+1+2 k \rho} Y^{-1} .
$$

Combining (4.12) and (4.14), we obtain

$$
\begin{aligned}
\left|b_{2} r_{1} h-b_{1} r_{2}\right| & =\left|r_{1} h\left(b_{2}-r_{2} d^{k} \alpha\right)+r_{2}\left(r_{1} h d^{k} \alpha-b_{1}\right)\right| \\
& \leq r_{1} h\left|r_{2} d^{k} \alpha-b_{2}\right|+r_{2}\left|r_{1} h d^{k} \alpha-b_{1}\right| \\
& \leq 1 / 2+2 x^{-k+2+4 k \rho} M^{-2}<1,
\end{aligned}
$$

whence

$$
\frac{b_{1}}{r_{1}}=\frac{h b_{2}}{r_{2}}, \quad r_{1}=\frac{r_{2}}{\left(r_{2}, h\right)} .
$$

We write $Z_{d}=Y X^{k-1} N_{d}^{k-1}\left|r_{2} d^{k} \alpha-b_{2}\right|$ and by Lemma 2, we get

$$
T_{4}(\alpha)=\sum_{(d, h) \in \mathcal{H}} \frac{y x^{\varepsilon} / d}{\left(r_{1}+Z_{d} h\right)^{1 / k}}\left(r_{2}, h\right)^{1 / k} \ll \sum_{d \leq y / M} \frac{y^{2} x^{\varepsilon} M^{-1}}{d^{2}\left(r_{2}+y(M d)^{-1} Z_{d}\right)^{1 / k}} .
$$

Hence

$$
T_{4}(\alpha) \ll y^{2-2 \rho+\varepsilon} M^{-1}+T_{5}(\alpha),
$$

where

$$
T_{5}(\alpha)=\sum_{d \in \mathcal{D}} \frac{y^{2} x^{\varepsilon} M^{-1}}{d^{2}\left(r_{2}+y(M d)^{-1} Z_{d}\right)^{1 / k}},
$$

and $\mathcal{D}$ is the set of integers $d \leq x^{2 \rho}$ for which there exist integers $b_{2}$ and $r_{2}$ with

$$
1 \leq r_{2} \leq x^{2 k \rho}, \quad\left(b_{2}, r_{2}\right)=1, \quad\left|r_{2} d^{k} \alpha-b_{2}\right| \leq y^{-2} x^{2-k+2 k \rho} .
$$

Combining (1.2), (1.4) and (4.16), we deduce that

$$
\begin{aligned}
\left|r_{2} d^{k} a-b_{2} q\right| & =\left|r_{2} d^{k}(a-q \alpha)+q\left(r_{2} d^{k} \alpha-b_{2}\right)\right| \\
& \leq r_{2} d^{k} Q^{-1}+q\left|r_{2} d^{k} \alpha-b_{2}\right| \\
& \leq x^{4 k \rho} Q^{-1}+y^{-2} x^{2-k+2 k \rho} Q<1,
\end{aligned}
$$

whence

$$
\frac{b_{2}}{r_{2}}=\frac{d^{k} a}{q}, \underset{10}{r_{2}}=\frac{q}{\left(q, d^{k}\right)}
$$


Thus, recalling Lemma 2, we get

$$
T_{5}(\alpha) \ll \frac{y^{2} x^{\varepsilon} M^{-1}}{\left(q+y^{2} x^{k-2}|q \alpha-a|\right)^{1 / k}} \sum_{d \leq x^{2 \rho}} \frac{\left(q, d^{k}\right)^{1 / k}}{d^{2}} \ll \frac{y^{2} x^{\varepsilon} M^{-1}}{\left(q+y^{2} x^{k-2}|q \alpha-a|\right)^{1 / k}} .
$$

The desired estimate follows from (4.3), (4.4), (4.5), (4.7), (4.11), (4.13), (4.15) and (4.17).

\section{Proof of Theorem 1}

In this section we deduce Theorem 1 from Propositions 1 and 2 and Vaughan's identity for $\Lambda(n)$.

Proof of Theorem 1. Let

$$
U=x^{\theta / 2-\rho}, \quad V=x^{1-\theta+2 \rho} .
$$

By (1.5), we have

$$
U V \asymp(x+y) / U \asymp x^{1-\theta / 2+\rho} \ll y^{1-2 \rho} .
$$

And then we apply Vaughan's identity (see [12]) in the following form

$$
\Lambda(n)=\sum_{\substack{m d=n \\ 1 \leq d \leq V}} \mu(d) \log m-\sum_{\substack{l m d=n \\ 1 \leq d \leq V \\ 1 \leq m \leq U}} \mu(d) \Lambda(m)-\sum_{\substack{l m d=n \\ 1 \leq d \leq V \\ m>U \\ l d>V}} \mu(d) \Lambda(m) .
$$

Thus we deduce that

$$
S_{k}(x, y ; \alpha)=S_{1}-S_{2}-S_{3},
$$

where

$$
\begin{aligned}
S_{1} & =\sum_{1 \leq d \leq V} \mu(d) \sum_{\substack{x<m d \leq x+y \\
S_{2}}}(\log m) e\left((m d)^{k} \alpha\right), \\
S_{3} & =\sum_{1 \leq v \leq U V} \lambda_{0}(v) \sum_{\substack{x<l v \leq x+y \\
V<u \leq(x+y) / U}} \lambda_{1}(u) \sum_{\substack{x<m u \leq x+y \\
m>U}} \Lambda(m) e\left((m u)^{k} \alpha\right),
\end{aligned}
$$

in which

$$
\lambda_{0}(v)=\sum_{\substack{m d=v \\ 1 \leq d \leq V \\ 1 \leq m \leq U}} \mu(d) \Lambda(m) \quad \text { and } \quad \lambda_{1}(u)=\sum_{\substack{d \mid u \\ 1 \leq d \leq V}} \mu(d) .
$$

We begin with estimating the sum $S_{3}$. Take

$$
\gamma=(\theta-3 / 4)^{-1}
$$

Since $3 / 4<\theta \leq 1$, by (1.5), we have

$$
\frac{1}{(1-2 \rho)} \frac{3 \gamma-\sigma_{k}-1}{2\left(2 \gamma-\sigma_{k}-1\right)} \leq \theta \leq 1
$$


To apply Proposition 2, we further divide $S_{3}$ into to two parts

$$
S_{31}=\sum_{x^{1 / 2} \leq u \leq(x+y) / U} \lambda_{1}(u) \sum_{\substack{x<m u \leq x+y \\ m>U}} \Lambda(m) e\left((m u)^{k} \alpha\right),
$$

and

$$
S_{32}=\sum_{V<u<x^{1 / 2}} \lambda_{1}(u) \sum_{\substack{x<m u \leq x+y \\ m>U}} \Lambda(m) e\left((m u)^{k} \alpha\right) .
$$

On noting that (5.1), (5.2) and $\lambda_{1}(u) \leq \tau(u)$, we can divide the summation over $u$ into dyadic intervals to deduce from Proposition 2 that

$$
\begin{aligned}
S_{31} & \ll(\log x) \max _{x^{1 / 2} \leq M \leq(x+y) / U}\left|\sum_{u \sim M} \xi(u) \sum_{x<m u \leq x+y} \eta(m) e\left((m u)^{k} \alpha\right)\right| \\
& \ll y^{1-\rho+\varepsilon}+\frac{y x^{\varepsilon}}{\left(q+y^{2} x^{k-2}|q \alpha-a|\right)^{1 /(2 k)}},
\end{aligned}
$$

where $\xi(u)=\lambda_{1}(u)$, and $\eta(m)=\Lambda(m)$ if $m>U$ and is 0 if else. For $S_{32}$, we first interchange the order of summation, and then by the same argument as above, we obtain

$$
S_{32} \ll y^{1-\rho+\varepsilon}+\frac{y x^{\varepsilon}}{\left(q+y^{2} x^{k-2}|q \alpha-a|\right)^{1 /(2 k)}} .
$$

Hence we get

$$
S_{3} \ll y^{1-\rho+\varepsilon}+\frac{y x^{\varepsilon}}{\left(q+y^{2} x^{k-2}|q \alpha-a|\right)^{1 /(2 k)}} .
$$

Next we estimate $S_{2}$. Write

$$
S_{4}(Z, W)=\sum_{Z<v \leq W} \lambda_{0}(v) \sum_{x<l v \leq x+y} e\left((l v)^{k} \alpha\right) .
$$

Then we find that

$$
S_{2}=S_{4}(0, V)+S_{4}(V, U V) .
$$

Note that (5.1), (5.2) and the bound $\left|\lambda_{0}(v)\right| \leq \log v$, we deduce from Proposition 2 that

$$
S_{4}(V, U V) \ll y^{1-\rho+\varepsilon}+\frac{y x^{\varepsilon}}{\left(q+y^{2} x^{k-2}|q \alpha-a|\right)^{1 /(2 k)}} .
$$

We then estimate $S_{4}(0, V)$. Since $3 / 4<\theta \leq 1$, by (1.5), (5.1) and (5.5), we have

$$
V \ll y\left(\frac{y}{x}\right)^{\frac{\gamma+1}{\gamma-\sigma_{k}-1}}, \quad V \ll y x^{-\gamma \rho / \sigma_{k}}, \quad V^{2 k} \ll y x^{k-1-2 k \rho} .
$$

So we can divide the summation over $v$ into dyadic intervals to deduce from Proposition 1 that

$$
S_{4}(0, V) \ll y^{1-\rho+\varepsilon}+\frac{y x^{\varepsilon}}{\underset{12}{\left(q+y x^{k-1}|q \alpha-a|\right)^{1 / k}} .}
$$


Thus, by combining (5.8) and (5.9), we deduce from (5.7) that

$$
S_{2} \ll y^{1-\rho+\varepsilon}+\frac{y x^{\varepsilon}}{\left(q+y^{2} x^{k-2}|q \alpha-a|\right)^{1 /(2 k)}} .
$$

Finally, in order to estimate $S_{1}$, we apply (3.4) directly, proceeding as in the treatment of $S_{4}(0, V)$. Thus we again obtain the bound

$$
S_{1} \ll y^{1-\rho+\varepsilon}+\frac{y x^{\varepsilon}}{\left(q+y^{2} x^{k-2}|q \alpha-a|\right)^{1 /(2 k)}} .
$$

Theorem 1 follows from (5.4), (5.6), (5.10) and (5.11).

\section{Proof of Theorem 2}

We outline our proof of Theorem 2, which proceeds via the circle method. Suppose that $k$ and $s$ are integers with $k \geq 2$ and $s \geq t_{k}$, where $t_{k}$ is defined as in (1.3). Let $\theta$ be a real number with $3 / 4<\theta<1$, and let $\delta$ be a sufficiently small, but fixed, positive number with $4 K \delta<\min \{\theta-3 / 4,1-\theta\}$. Consider a sufficiently large natural number $N$, put $X=(N / s)^{1 / k}$, and write $Y=X^{\theta}$. When $n$ is a natural number with $N \leq n \leq N+X^{k-1} Y$, we denote

$$
\rho_{s}(n)=\sum_{\left|p_{1}-X\right| \leq Y} \cdots \sum_{\left|p_{s}-X\right| \leq Y}\left(\log p_{1}\right) \cdots\left(\log p_{s}\right)
$$

which is the weighted number of solutions of the equation (1.7) with $\left|p_{i}-X\right| \leq Y$ (1 $\leq$ $i \leq s)$. Define

$$
f(\alpha)=\sum_{\substack{|p-X| \leq Y \\ p \text { prime }}}(\log p) e\left(p^{k} \alpha\right)
$$

Then it follows from orthogonality that

$$
\rho_{s}(n)=\int_{0}^{1} f(\alpha)^{s} e(-n \alpha) d \alpha .
$$

Next we define the Hardy-Littlewood dissection. We rewrite $P$ and $Q$ to be

$$
P=X^{2 K \delta}, \quad Q=X^{k-2} Y^{2} P^{-1} .
$$

Then let $\mathfrak{M}$ and $\mathfrak{m}$ be the major arc and minor arc as in $₫ \mathbb{1}$, respectively, with $P$ and $Q$ defined above. When $\mathfrak{B}$ is a measurable subset of $[0,1)$, we define

$$
\rho_{s}(n ; \mathfrak{B})=\int_{\mathfrak{B}} f(\alpha)^{s} e(-n \alpha) d \alpha .
$$

Thus, since $[0,1)$ is the disjoint union of $\mathfrak{M}$ and $\mathfrak{m}$, we find from (6.2) that

$$
\rho_{s}(n)=\rho_{s}(n ; \mathfrak{M})+\rho_{s}(n ; \mathfrak{m}) .
$$

The major arc contribution can be summarised in the following proposition. 
Proposition 3. Suppose that $k \geq 2$ and $s \geq \min \{5, k+2\}$. Then, whenever $19 / 24<$ $\theta<1, Y=X^{\theta}$ and $n$ is a natural number with $N \leq n \leq N+X^{k-1} Y$, we have

$$
\rho_{s}(n ; \mathfrak{M})=\mathfrak{S}(n) \mathfrak{J}(n)+O\left(Y^{s-1} X^{1-k}(\log X)^{-1}\right),
$$

where the singular integral

$$
\mathfrak{J}(n)=\int_{0}^{1} v(\beta)^{s} e(-\beta n) d \beta
$$

with

$$
v(\beta)=k^{-1} \sum_{(X-Y)^{k} \leq m \leq(X+Y)^{k}} m^{-1+1 / k} e(\beta m),
$$

and the singular series

$$
\mathfrak{S}(n)=\sum_{q=1}^{\infty} \varphi(q)^{-s} \sum_{\substack{a=1 \\(a, q)=1}}^{q} S(q, a)^{s} e(-n a / q) .
$$

with

$$
S(q, a)=\sum_{\substack{r=1 \\(q, r)=1}}^{q} e\left(a r^{k} / q\right)
$$

Moreover, we have

$$
Y^{s-1} X^{1-k} \ll \mathfrak{S}(n) \mathfrak{J}(n) \ll Y^{s-1} X^{1-k}(\log X)^{\eta},
$$

where $\eta=\eta(s, k)$ is a positive number.

Proof. See [14, Proposition 2.1 and eq. (2.7)].

In order to estimate the minor arc contribution, we have the following analogue of Hua's lemma.

Proposition 4. Suppose that $y$ is a real number with $y \geq x^{1 / 2}$. Then whenever $s \geq 2 t_{k}$ and $\varepsilon>0$, we have

$$
\int_{0}^{1}|f(\alpha)|^{s} d \alpha \ll y^{s-1} x^{1-k+\varepsilon} .
$$

Proof. See [14, Proposition 2.2].

Next, by Theorem 1, we establish a non-trivial estimate for $f(\alpha)$ throughout the set of minor arcs $\mathfrak{m}$.

Proposition 5. Let $k \geq 3$. Let $\theta$ be a real number with $3 / 4<\theta \leq 1$ and suppose that

$$
\varrho=\varrho_{k}(\theta)=\frac{1}{2} \min \left\{\frac{\sigma_{k}(\theta-3 / 4)}{8}, \delta\right\},
$$

where $\sigma_{k}=1 /\left(2 t_{k}\right)$. Then, for any fixed $\varepsilon>0$, we have

$$
\max _{\alpha \in \mathfrak{m}}|f(\alpha)| \ll Y^{1-\varrho+\varepsilon} .
$$


Proof. Take $x=X-Y, y=2 Y$. Recall that for $\alpha \in \mathfrak{m}$, we have $q>P$. Proposition 5 is an easy consequence of Theorem 1 .

Proof of Theorem 2. Now following the same argument as in [14, §2], we give the proof of Theorem 2 by combining Propositions 3 , 4 and 5.

Acknowledgement. The author would like to thank Professor Jianya Liu for his valuable advice and constant encouragement. The author also want to thank Bin Wei for explaining many details in their paper.

\section{REFERENCES}

1. D. Daemen, The asymptotic formula for localized solutions in Waring's problem and approximations to Weyl sums, Bull. Lond. Math. Soc. 42 (2010), no. 1, 75-82. MR 2586968 (2011b:11139)

2. B. R. Huang, Strong orthogonality between the Möbius function and nonlinear exponential functions in short intervals, Int. Math. Res. Not. (2015), rnv091.

3. B. R. Huang and Z. W. Wang, Exponential sums over primes in short intervals, Journal of Number Theory 148 (2015), 204-219.

4. M. N. Huxley, On the difference between consecutive primes, Invent. Math. 15 (1972), 164-170. MR 0292774 (45 \#1856)

5. K. Kawada and T. D. Wooley, On the Waring-Goldbach problem for fourth and fifth powers, Proceedings of the London Mathematical Society 83 (2001), no. 1, 1-50.

6. A. V. Kumchev, On Weyl sums over primes and almost primes, Michigan Math. J 54 (2006), no. 2, $243-268$.

7. _ On Weyl sums over primes in short intervals, Number theory - arithmetic in Shangri-La, Ser. Number Theory Appl., vol. 8, World Sci. Publ., Hackensack, NJ, 2013, pp. 116-131.

8. J. Y. Liu, G. S. Lü, and T. Zhan, Exponential sums over primes in short intervals, Science in China Series A 49 (2006), no. 5, 611-619.

9. J. Y. Liu and T. Zhan, Estimation of exponential sums over primes in short intervals II, Analytic Number Theory: Proceedings of a Conference in Honor of Heini Halberstam, Birkhauser, 1996, pp. 571-606.

10. _ Estimation of exponential sums over primes in short intervals I, Monatshefte für Mathematik 127 (1999), no. 1, 27-41.

11. G. S. Lü and H. X. Lao, On exponential sums over primes in short intervals, Monatshefte für Mathematik 151 (2007), no. 2, 153-164.

12. R. C. Vaughan, Recent work in additive prime number theory, Proceedings of the International Congress of Mathematicians (Helsinki, 1978), Acad. Sci. Fennica, Helsinki, 1980, pp. 389-394. MR 562631 (81j:10077)

13. I. M. Vinogradov, Estimation of certain trigonometric sums with prime variables, Izv Acada Nauk SSSR 3 (1939), 371-398.

14. B. Wei and T. D. Wooley, On sums of powers of almost equal primes, Proc. London Math. Soc. (to appear), 38pp. (2014).

15. T. Zhan, On the representation of large odd integer as a sum of three almost equal primes, Acta Mathematica Sinica 7 (1991), no. 3, 259-272.

School of Mathematics, Shandong University, Jinan, Shandong 250100, China

E-mail address: brhuang@mail.sdu.edu.cn 